\section{Tribulus terrestris protects rat myocardium against isoproterenol-induced ischemic injury: role of HSP 70 and cardiac endogenous antioxidants}

\author{
Ipseeta Ray Mohanty, 1 \\ Ujjwala Maheshwari,2 Daniel Joseph,1 \\ Yeswant Deshmukh1 \\ 1Department of Pharmacology; \\ 2Department of Pathology, MGM Medical \\ College, Navi Mumbai, India
}

\section{Abstract}

The present study was undertaken to evaluate the cardioprotective activity of Tribulus terrestris (Tt), a medicinal herb following isoproterenol (ISP)-induced myocardial injury. The contribution of heat shock protein (HSP) 70, key anti-stress protein, endogenous antioxidants and oxidant -antioxidant balance in attenuating myocardial injury was further studied. Hydroalcoholic extract of $\mathrm{Tt}\{1,2.5,5$ \& $10 \mathrm{mg} / \mathrm{kg}$ \} were orally fed once a daily to Wistar rats for 21 days. On the 20 th and $21^{\text {st }}$ day, both control (ISP control) and Tt fed rats were challenged with ISP $(85 \mathrm{mg} / \mathrm{kg}$, s. c. two doses at $24 \mathrm{~h}$ intervals) induced myocardial necrosis. Histopathological evaluation, cardiac marker enzyme: Creatinine phosphokinase(CPK) and antioxidative parameters: Glutathione (GSH), Thiobarbituric acid reactive substances (TBARS), Catalase (CAT), Glutathione peroxidase (GSHPx) and Superoxide dismutase (SOD) levels were estimated. Tt $(2.5 \mathrm{mg} / \mathrm{kg})$ intake per se upregulated HSP 70; increased basal SOD, CAT activity $(\mathrm{P}<0.05)$ and caused a marked fall in basal TBARS levels $(\mathrm{P}<0.05)$ in comparison to sham. Following ISP challenge, significant oxidative stress with evidence of myocardial necrosis was observed in the ISP control group. ISPinduced changes in myocardial SOD, GSHPx and GSH were prevented by both the 2.5 and 10 $\mathrm{mg} / \mathrm{kg}$ doses of $\mathrm{Tt}$, though cellular injury was minimal with $2.5 \mathrm{mg} / \mathrm{kg}$ dose. The results emphasize that pre-treatment with Tt offered significant protection against ISP-induced myocardial necrosis through a unique property of enhancement of endogenous antioxidants, stabilization of cytoskeleton structure which in turn is attributed to HSP 70 expression along with fortified antioxidant defense system.

\section{Introduction}

Oxidative stress is the major etiopathologi- cal factor in ISP-induced myocardial necrosis. A relatively low amount of endogenous antioxidant makes the heart vulnerable to oxidative stress-induced damage. ${ }^{1-3}$ Considerable efforts have been made in the exploration of the potential for exogenous antioxidants and free radical scavengers to supplement endogenous antioxidant system and limit free radical injury, with mixed success and failure. ${ }^{4,5}$ One reason why exogenous antioxidants have limited success in the prevention of myocardial injury may be due to the inaccessibility of large molecules to the key intracellular sites of oxidative damage. Under such circumstances, other options need to be explored which will help in circumventing this problem, such as, whether, by any means, it is possible to stimulate or augment the endogenous antioxidant defense system of the heart. 5

A concept is now emerging of 'adaptogenic drugs' drugs that increase non-specific resistance of the users to a variety of stresses. One of the proposed mechanisms of action of such drugs is by enhancing basal cellular endogenous antioxidant enzymes (SOD, CAT, GSHPx), and nucleic acid biosynthesis. ${ }^{6,7}$ Role of heat shock proteins (HSPs), most notably the inducible $70-\mathrm{kDa}$ HSP family member HSP 70 , in myocardial adaptation has been well documented. HSP 70 is believed to participate in the protection of the myocardium during cardiovascular stress as well as apoptosis. Up regulation of HSP70 has been associated with the reduction in myocardial apoptosis as it prevents the degradation of nucleolin, an antiapoptotic protein. ${ }^{8}$ In addition, studies demonstrated that HSP 70 enhanced the mitochondrial energetic capacity and increased the tolerance to myocardial injury. 9,10 This unique property of augmenting endogenous antioxidants and HSP 70 has been explored in the present study. ${ }^{5}$

Tribulus terrestris $(\mathrm{Tt})$ has long been a constituent in tonics in Indian ayurveda practice, where it is known by its Sanskrit name, gokshura. It is also used as an aphrodisiac, diuretic and nervine in Ayurveda, and in Unani, another medical system of India. The saponins (furostanol) and flavanoids present in the leaves and fruits are the active principles responsible for its vasodilatory and diuretic properties. ${ }^{10,11}$ Since the first report of cardiac actions of Tt was published in 1976,12 there has been a paucity of research investigating the potential benefits of using $\mathrm{Tt}$ as a cardioprotective agent. Recently, Guo et al., evaluated the effects of Tt saponins on ventricular remodeling after myocardial infarction in hyperlipidemic rats. ${ }^{13}$ However, the cardioprotective and adaptogenic potential of $\mathrm{Tt}$ in the setting of ISP induced myocardial necrosis has not been studied before. By virtue of its adaptogenic and antioxidant properties, Tt may offer protection against oxidative stress-
Correspondence: Ipseeta Ray Mohanty, Department of Pharmacology, MGM Medical College, Kamothe, Navi Mumbai, Maharasthra, 410209, India.

Tel. +91.9819908498 - Fax: +91.2256661988 .

E-mail: ipseetamohanty@yahoo.co.in

Key words: Oxidative stress, Myocardial infarction, Medicinal herbs, Tribulus terrestris, Antioxidants.

Acknowledgements: the authors gratefully acknowledge the financial assistance (Grant No: SR/FT/L-124/2005) from the Department of Science and Technology (DST), Government of India for conducting the study.

Contributions: IRM, study design and study conduction, study results tabulation and analysis; UM, histopathological and immunohistochemical study, pathological results interpretation; DJ, YD, study results performing and interpretation, manuscript refining.

Conflict of interest: the authors report no conflicts of interest.

Received for publication: 1 June 2011. Accepted for publication: 3 August 2011.

This work is licensed under a Creative Commons Attribution NonCommercial 3.0 License (CC BYNC 3.0).

(C) Copyright I.R. Mohanty et al., 2011 Licensee PAGEPress, Italy

Alternative Medicine Studies 2011; 1:e9

doi:10.4081/ams.2011.e9

induced by ISP, a catecholamine known to have deleterious effects on the myocardium. ${ }^{5}$ With this point of view the study was designed to provide scientific data and validate the cardioprotective effects of $\mathrm{Tt}$ as well as to delineate it mechanism of action.

Immunohistochemical localization of HSP 70 , indices of oxidant-antioxidant balance: Thiobarbituric acid reactive substances (TBARS), endogenous antioxidant: GSH and antioxidant enzymes (SOD, CAT, GSHPx) were incorporated into the study design. Cardioprotection was confirmed by assessing the severity of pathological changes and myocardial CPK.

\section{Materials and methods}

\section{Experimental animals}

Adult male Wistar rats, 10 to 12 weeks old, weighing 150 to $200 \mathrm{~g}$ were used in the study. The study protocol was reviewed and approved by the Institutional Animal Ethics Committee and conforms to the Indian National Science 
Academy Guidelines for the Use and Care of Experimental Animals in Research. Animals were obtained from the Animal Facility of Mahatma Gandhi Mission Medical College, Navi Mumbai, India. The rats were housed in an air-conditioned room and were kept in standard laboratory conditions under natural light and dark cycles (approximately $14 \mathrm{~h}$ light/10 h dark) and maintained at humidity $60 \pm 5 \%$ and an ambient temperature of $25 \pm 2{ }^{\circ} \mathrm{C}$. The animals were allowed free excess to standard diet and tap water ad libitum and allowed to acclimatize for one week before the experiments.

\section{Chemicals}

All Chemicals were of analytical grade, purchased from Sigma Chemical Co., St Louis, USA. The ABC staining kit and secondary antibodies (Anti mouse IgG) were procured from Santa Cruz Biotechnology, USA. HSP 70 mouse monoclonal IgG1 primary antibody was procured from Biogenex Life Sciences Private limited, India.

Hydro-alcoholic lyophilized extracts of Tribulus terrestris was procured from Dabur Research Foundation, India. The multiple solvent (methanol: isopropyl alcohol: acetone) extraction procedure was used to prepare the extract by the supplier. The whole plant was used for extraction. The extractive values (taking $1 \mathrm{gm}$ sample) in water was $64.15 \% \mathrm{w} / \mathrm{w}$ and in methanol was $53.94 \mathrm{w} / \mathrm{w}$. pH of 1\% w/v aqueous solution was 8.23 and loss on drying value at $105^{\circ} \mathrm{C}$ by infrared balance was $3.1 \mathrm{w} / \mathrm{w}$. The total ash content was $14.06 \%$ w/w. Total saponin content (on dried basis) was not less than $20-45 \%$ w/w by HPLC.

\section{Experimental groups and treatment protocol}

The animals were assigned to the following experimental groups. There were ten animals in each group.

\section{Baseline evaluation protocol}

In this group healthy experimental animals were used to evaluate baseline values of various parameters investigated in this study i.e rats without any pathologic challenge to the heart

\section{Group 1 - Saline control group (Sham)}

Rats were administered $0.9 \%$ normal saline per orally using a feeding cannula for 21 days and then sacrificed on the 22nd day.

\section{Group 2 -Tribulus terrestris control group}

This group was divided in four subgroups. Hydro-alcoholic extract of Tt was dissolved w/v in $0.9 \%$ normal saline administered orally to healthy experimental rats once daily for 21 days at the doses $1 \mathrm{mg} / \mathrm{kg}$ (Tt-1), $2.5 \mathrm{mg} / \mathrm{kg}$ (Tt2.5), $5 \mathrm{mg} / \mathrm{kg}$ (Tt-5) and $10 \mathrm{mg} / \mathrm{kg}$ (Tt-10).

\section{Isoproterenol (ISP)-induced} myocardial necrosis protocol

Group 3 - Isoproterenol group - ISP control

The rats were administered $0.9 \%$ normal saline for 21 days and in addition administered ISP ( $85 \mathrm{mg} / \mathrm{kg}$, subcutaneously) on 20th and 21 st day at an interval of $24 \mathrm{~h}$.

\section{Group 4 - Tribulus terrestris treated group}

Tt was administered orally to healthy experimental rats once daily for 21 days and thereafter, the rats, were challenged with ISP (85 $\mathrm{mg} / \mathrm{kg}$ ) on $20^{\text {th }}$ and $21 \mathrm{st}$ day at an interval of 24 h. This group was further divided into three subgroups:

Group 4a -ISP + $1 \mathrm{mg} / \mathrm{kg}$ (ITt-1)

Group 4b - ISP + $2.5 \mathrm{mg} / \mathrm{kg}$ (ITt-2.5)

Group $4 \mathrm{c}$ - ISP $+5 \mathrm{mg} / \mathrm{kg}$ (ITt-5)

Group 4d - ISP + 10mg/kg (ITt-10)

\section{Experimental parameters studied}

\section{Biochemical studies}

A ten-percent homogenate of myocardial tissue was prepared in $50 \mathrm{mM}$ phosphate buffer, pH 7.4 and an aliquot was used for the assay of TBARS according to the method described by Ohkawa et al,.14 The homogenate was centrifuged at $7000 \mathrm{rpm}$ for 15 minutes and the supernatant was used for the estimation of the glutathione, ${ }^{15}$ glutathione peroxidase, ${ }^{16}$ superoxide dismutase, ${ }^{17}$ Catalase, ${ }^{18}$ and protein. ${ }^{19}$ Creatinine phosphokinase was estimated spectrophotometrically using a kit from Randox Laboratories, USA. ${ }^{20}$

Immunostaining for the Localization of HSP Proteins

Immunohistochemistry using the mouse anti-cleaved monoclonal heat shock protein IgG1 antibody was performed on deparaffinized tissue sections using a routine avidin-biotin-immunoperoxidase "technique". Before incubation with the primary rabbit polyclonal antibody (1:1000 dilution), tissue sections were subjected to heat-induced epitome retrieval by incubation in a $\mathrm{pH} 8.0$ $0.01 \mathrm{M}$ EDTA solution for $10 \mathrm{~min}$ in a vegetable steamer, followed by 20 -min cool-down and treatment with $3 \%$ hydrogen peroxide before antibody application. Bound antibodies were detected using HRP-Straptavidin complex. The target protein (HSP) was visualized by incubation in peroxidase substrate complex and DAB (3,3' diaminobenzidine) as the chromogen. Counterstaining was performed with Meyer's hematoxylin. All descriptions and the pictures given in the manuscript are based on specific staining as adjusted against the positive and negative controls.
Histopathological studies

At the end of the experiment, myocardial tissue was immediately fixed in $10 \%$ buffered neutral formalin solution. Cross sections (5 $\mu \mathrm{m}$ thick) of the fixed myocardial tissues were cut. These sections were stained with hematoxylin and eosin (H\&E) and visualized under light microscope to study the light microscopic architecture of the myocardium. The degree of necrosis was graded and scored.

\section{Statistical analysis}

All numerical data in text, figures and tables are expressed as the mean + SD. Statistical analysis was performed by one-way analysis of variance (ANOVA) or repeated measures ANOVA, followed by the Bonferroni post hoc test. Differences were considered statistically significant at $\mathrm{P}<0.05$.

\section{Results}

\section{Isoproterenol (ISP)-induced myocardial necrosis protocol}

Biochemical parameters without ISPinduced myocardial necrosis

Oral feeding of Tt (2.5 and $10 \mathrm{mg} / \mathrm{kg}$ ) per se to healthy experimental rats for 21 days, augmented basal SOD and CAT activity $(\mathrm{P}<0.05)$ as compared to sham baseline values (Table 1). Tt at 1 , $5 \& 10 \mathrm{mg} / \mathrm{kg}$ did not alter basal TBARS levels; only $2.5 \mathrm{mg} / \mathrm{kg}$ dose was able to significantly reduce baseline lipid peroxidation as evidenced by fall in basal TBARS level $(\mathrm{P}<0.05)$ in comparison to sham. In addition, no significant increase in the basal GSH levels and GSHPx activity was seen in any of the Tt control groups in reference to sham group (Table 1).

\section{Biochemical parameters following \\ ISP-induced myocardial necrosis}

ISP induced myocardial necrosis resulted in a significant depletion of myocardial GSH $(\mathrm{P}<0.05)$, fall in myocardial antioxidant enzymes $\{$ SOD $(\mathrm{P}<0.01)$, CAT $(\mathrm{P}<0.01)$, GSHPx $(\mathrm{P}<0.05), \mathrm{CPK}(\mathrm{P}<0.01)\}$ and increase in lipid peroxidation as evidenced by elevated \{TBARS levels $(\mathrm{P}<0.01)\}$ as compared to sham (Table 2). Tt (2.5 and $10 \mathrm{mg} / \mathrm{kg}$ ) doses significantly inhibited lipid peroxidation ( $\mathrm{P}<0.05$, Figure 1 ) and preserved membrane integrity. In addition, significant restoration of myocardial $\mathrm{CPK}$ enzyme with $\mathrm{Tt}-2.5 \mathrm{mg} / \mathrm{kg}(\mathrm{P}<0.01)$ and $\mathrm{Tt}-10$ $(\mathrm{P}<0.05)$ was observed in reference to ISP control. Subsequent to ISP induced myocardial necrosis, Tt(2.5 and $10 \mathrm{mg} / \mathrm{kg}$ ) significantly protected SOD $(\mathrm{P}<0.05)$ and $\operatorname{GSHPx}(\mathrm{P}<0.05)$ (Figure 2). However, none of the doses studied restored GSH levels as compared to ISP control (Table 2). 
Heat shock protein 70 expression following Tt pre-treatment

HSP70 immunostaining in the myocardium was very faint in sham operated controls $(4.2+4.2 \%$; Figure $3 \mathrm{~A})$. In the $\mathrm{Tt}(1 \mathrm{mg} / \mathrm{kg}$ and $5 \mathrm{mg} / \mathrm{kg}$ ) control group no significant change in the expression of basal HSP 70 $(6.2+2.1 \% ; 4.8+3.1 \%)$ respectively was observed as compared to sham group. In both the groups, myocytes were weakly positive for HSP70. However, in the Tt $(2.5 \mathrm{mg} / \mathrm{kg})$ control group oral pretreatment for 21 day resulted in significant up-regulation $(35.2+$ $3.1 \%$ ) of basal HSP 70 expression (Figure $3 \mathrm{~B})$. Similarly, in the Tt $(10 \mathrm{mg} / \mathrm{kg})$ control group strong positive staining was seen in microvessels as well as myocytes $(25.2+3.1 \%)$ as compared to sham group (Figure $3 \mathrm{C}$ ).

\section{Histopathological studies following} ISP-induced myocardial necrosis

Microscopic histology revealed that the sham group was characterized by an organised pattern and shows normal architecture of the myocardium.

However, in the rat sections of the ISP control group, myonecrosis with fibroblastic proliferation, infiltration of inflammatory cells, marked intramyocellular edema, besides vacoular degeneration and rounded nuclei as compared to sham-operated group was observed. $\operatorname{Tt}(2.5$ and $10 \mathrm{mg} / \mathrm{kg})$ treatment prevented myonecrosis, infiltration of inflammatory cells, edema and vacuolar changes as compared to the ISP control. However, in the Tt- 1 and Tt-5 treated groups of the study protocol the degree of edema and necrosis was nearly comparable to that of ISP control group with similar morphological changes (Table 3 ).

\section{Discussion}

Nature has been a source of medicinal treatments for thousands of years and plant-derived products continue to play an essential role in the primary health care of about $80-85 \%$ of the world's population. ${ }^{21,22}$ There has been an upsurge in the use of medicinal plants for the

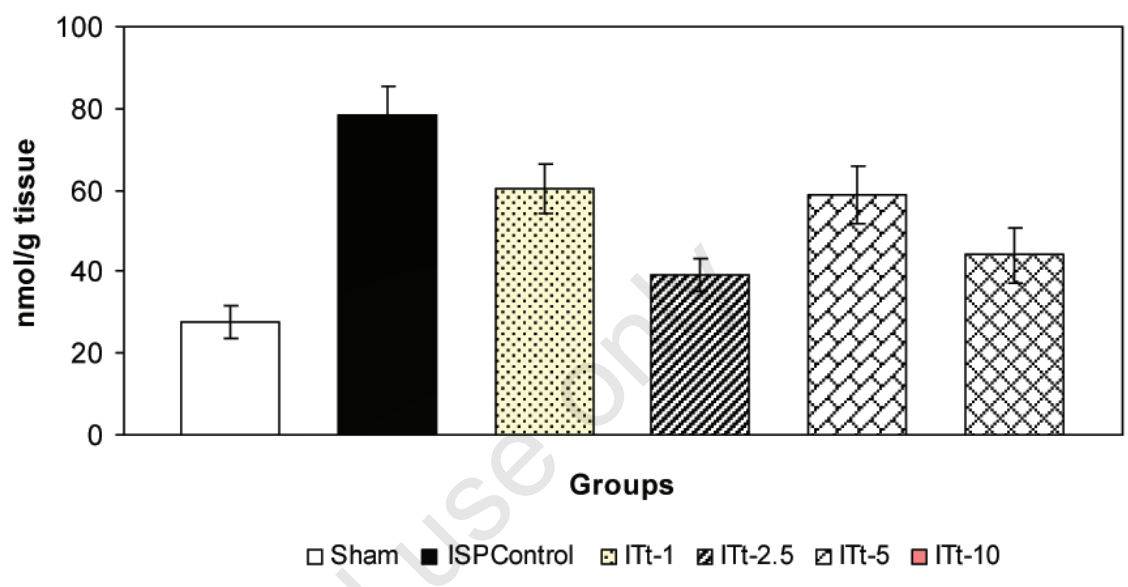

Figure 1. Myocardial TBARS levels in the different experimental groups. \#\#P<0.01 Vs Sham, ${ }^{*} \mathbf{P}<0.05$ Vs ISP Control.

Table 1. Biochemical parameters in the different groups.

\begin{tabular}{|c|c|c|c|c|c|}
\hline & Sham & Tt-1 & Tt-2.5 & Tt-5 & Tt-10 \\
\hline $\begin{array}{l}\text { GSH } \\
\text { (umol/g tissue) }\end{array}$ & $4.03 \pm 0.3$ & $4.4 \pm 0.6$ & $5.4 \pm 0.6$ & $4.23 \pm 0.4$ & $4.16 \pm 0.2$ \\
\hline $\begin{array}{l}\text { TBARS } \\
\text { (nmol/g tissue) }\end{array}$ & $29.73 \pm 5.7$ & $27.48 \pm 3.4$ & $18.33 \pm 1.3^{*}$ & $25.48 \pm 3.4$ & $22.8 \pm 0.9$ \\
\hline $\begin{array}{l}\text { GSHPx } \\
\text { (IU/mg protein) }\end{array}$ & $0.33 \pm 0.1$ & $0.38 \pm 0.1$ & $0.38 \pm 0.1$ & $0.41 \pm 0.1$ & $0.43 \pm 0.1$ \\
\hline $\begin{array}{l}\text { SOD } \\
\text { (IU/mg protein) }\end{array}$ & $7.94 \pm 2.9$ & $7.12 \pm 0.4$ & $28.04 \pm 1.2^{* *}$ & $7.12 \pm 0.4$ & $18.37 \pm 1.7^{* *}$ \\
\hline $\begin{array}{l}\text { CAT } \\
\text { (IU/mg protein) }\end{array}$ & $21.1 \pm 3.1$ & $26.0 \pm 5.6$ & $54.8 \pm 8.0^{*}$ & $26.0 \pm 5.6$ & $46.3 \pm 9.6^{*}$ \\
\hline $\begin{array}{l}\text { CPK } \\
\text { (IU/mg protein) }\end{array}$ & $6.7 \pm 1.4$ & $7.7 \pm 0.8$ & $7.3 \pm 0.8$ & $7.1 \pm 0.4$ & $7.4 \pm 0.5$ \\
\hline
\end{tabular}

${ }^{*} \mathrm{P}<0.05,{ }^{*} \mathrm{P}<0.01$ vs sham group. GSH(Glutathione);TBARS(Thiobarbituric acid reactive substances) \& Glutathione peroxidase(GSHPx), Superoxide dismutase(SOD), Catalase(CAT) and CPK(Creatinine phosphokinase). One unit of catalase activity represents $1 \mathrm{~mol}$ of $\mathrm{H}_{2} \mathrm{O}_{2}$ decomposed / min. One unit of SOD is defined as the amount of enzyme required to produce $50 \%$ inhibition of epinephrine. One unit of enzyme activity is defined as $1 \mathrm{nmol}$ of NADPH utilized per min at $37^{\circ} \mathrm{C}$. One unit of $\mathrm{CPK}$ is defined as the amount of enzyme that will transfer $1 \mu \mathrm{mol}$ of phosphate from phosphocreatine to $\mathrm{ADP}$ per min at pH 7.4 at $30^{\circ} \mathrm{C}$.

Table 2. Biochemical parameters in the different groups.

\begin{tabular}{lcccc}
$\begin{array}{l}\text { Groups/Biochemical } \\
\text { Parameters (units) }\end{array}$ & $\begin{array}{c}\text { SOD } \\
\text { (IU/mg protein) }\end{array}$ & $\begin{array}{c}\text { CAT } \\
\text { (IU/mg protein) }\end{array}$ & $\begin{array}{c}\text { GSHPx } \\
\text { (IU/mg protein) }\end{array}$ & $\begin{array}{c}\text { GSH } \\
\text { (umol/g tissue) }\end{array}$ \\
Sham & $7.94 \pm 1.93$ & $21.06 \pm 3.05$ & $0.33 \pm 0.09$ & $4.03 \pm 0.3$ \\
ISP Control & $3.36 \pm 0.97 \# \#$ & $11.92 \pm 1.98^{\# \#}$ & $0.19 \pm 0.08 \#$ & $2.3 \pm 0.1^{\#}$ \\
\hline ITt-1 & $4.01 \pm 0.42$ & $14.05 \pm 6.36$ & $0.22 \pm 0.03$ & $2.6 \pm 0.3$ \\
ITt-2.5 & $5.51 \pm 1.24^{*}$ & $16.05 \pm 6.36$ & $0.38 \pm 0.05^{*}$ & $3.02 \pm 0.1$ \\
\hline ITt-5 & $4.01 \pm 0.42$ & $15.43 \pm 4.91$ & $0.26 \pm 0.03$ & $2.8 \pm 0.2$ \\
ITt-10 & $5.75 \pm 1.29^{*}$ & $15.61 \pm 6.70$ & $0.29 \pm 0.10$ & $2.99 \pm 0.4$ \\
\hline
\end{tabular}

\#P $<0.01$ vs Sham; ${ }^{*} \mathrm{P}<0.05$ vs ISP Control. Glutathione peroxidase (GSHPx), Superoxide dismutase (SOD) and Catalase(CAT). One unit of catalase activity represents $1 \mu$ mol of $\mathrm{H}_{2} \mathrm{O}_{2}$ decomposed $/$ min. One unit of SOD is defined as the amount of enzyme required to produce $50 \%$ inhibition of epinephrine. One unit of enzyme activity is defined as $1 \mathrm{nmol}$ of NADPH utilized per min at $37^{\circ} \mathrm{C}$. 
treatment of various diseases. A concept is now emerging of adaptogenic drugs drugs that increase non-specific resistance of the users to a variety of stresses. Adaptogenic' property of various herbs like Ocimum sanctum, Bacopa monniera and Withania somnifera, first time reported by Brekhman and associates in Eleuthrococcus and Panax ginseng has already been reported in various experimental studies. 8,23 These herbs allow one to adapt to a variety of heightened stressful circumstances. Although the exact mechanism of such adaptation is presently not known, it has been proposed that these drugs may act by inducing a number of antioxidant enzymes such as SOD, CAT, GSHPx and antioxidants such as GSH, proteins HSP in the heart.7,24 The study was designed to evaluate the a) adaptogenic effects of $\mathrm{Tt}$ (based on modulation of myocardial antioxidant system and HSP 70 expression) and b) cardioprotective potential of the medicinal herb following ISP induced myocardial necrosis.

The present study for the first time demonstrates the adaptogenic property of Tt. Oral administration of Tt per se to healthy experimental animals resulted in a significant increase in CAT, SOD activity with 2.5 and 10 $\mathrm{mg} / \mathrm{kg}$ doses and inhibition of basal lipid peroxidation with $\mathrm{Tt}(2.5 \mathrm{mg} / \mathrm{kg})$ doses. However, none of the doses of Tt evaluated in this study resulted in significant augmentation of myocardial GSH content and GSHPx activity. Any increase in SOD activity is beneficial in the event of increased free radical generation. ${ }^{23}$ However, it has been reported that an augmented SOD activity, without a concomitant rise in the activity of CAT and/or GSHPx might be detrimental, since SOD activity, generates hydrogen peroxide as a metabolite, which is more cytotoxic than oxygen radicals and must be scavenged by CAT or GSHPx. A simultaneous increase in CAT and/or GSHPx activity is essential for an overall beneficial effect of an increased SOD activity. 25 Thus, simultaneous increase in myocardial SOD and CAT activities observed in the present study with administration of Tt may contribute to the adaptogenic effects of Tt pretreatment.

HSPs constitute an endogenous stress response for myocardial adaptation. ${ }^{26}$ There is mounting evidence that cardiac cells synthesize HSPs in response to a variety of stresses and that this stress response may result in protection from subsequent ischemic exposure. For example, several investigators have demonstrated increased cardiac HSP synthesis in response to hyperthermia, hemodynamic overload, cardiac transplantation hypoxia, and ischemia. $8,9,27$ These cytoprotective effects of HSPs make them tempting targets for therapeutic interventions and myocardial adaptation. HSP induction can be achieved by a multitude of different stimuli, among them hyper- thermia, hypoxia, ischemia, oxidative stress, and various drugs. ${ }^{8}$

In the present study, a nine-six fold increase in the basal expression of HSP 70 was observed in the Tt $(2.5$ and $10 \mathrm{mg} / \mathrm{kg}$ ) control groups. HSP 70 in the heart was exclusively expressed in myocytes and endothelial cells. Within these cells HSP 70 was co-localized in the nucleus and in the cytosol. These observations are analogous with those of other investigators concerning the apparent role of HSPs in protecting myocardial cells from stress as well as apoptosis. ${ }^{28}$ Upregulation of HSP70 is associated with the reduction in myocardial apoptosis as it prevents the degradation of

Table 3. Light microscopic changes in the different experimental groups.

\begin{tabular}{lccc} 
Groups & Necrosis & Edema & Inflammation \\
Sham & - & - & - \\
ISP Control & ++++ & +++ & +++ \\
\hline ITt-1 & ++++ & +++ & +++ \\
ITt-2.5 & ++ & ++ & + \\
\hline ITt-5 & +++ & ++ & +++ \\
ITt-10 & ++ & ++ & ++ \\
\hline
\end{tabular}

The degree of necrosis was graded and scored as follows: Score (-): Absence of any inflammation, edema and necrosis; Score (+): Focal areas of inflammation, edema and necrosis; Score $(++)$ : Patchy areas of inflammation, edema and necrosis; Score (+++): Confluent areas of inflammation, edema and necrosis; Score (++++): Massive areas of inflammation, edema and necrosis

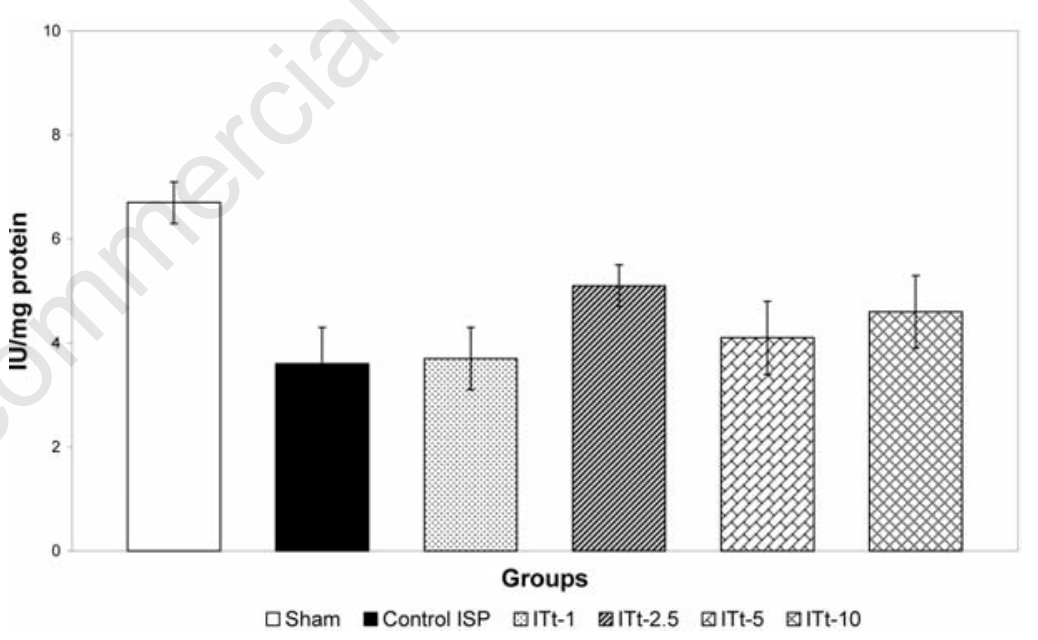

Figure 2. Myocardial CPK activity in the different experimental groups. \#\#P<0.01Vs Sham, ${ }^{*} \mathbf{P}<0.05$ Vs ISP Control.

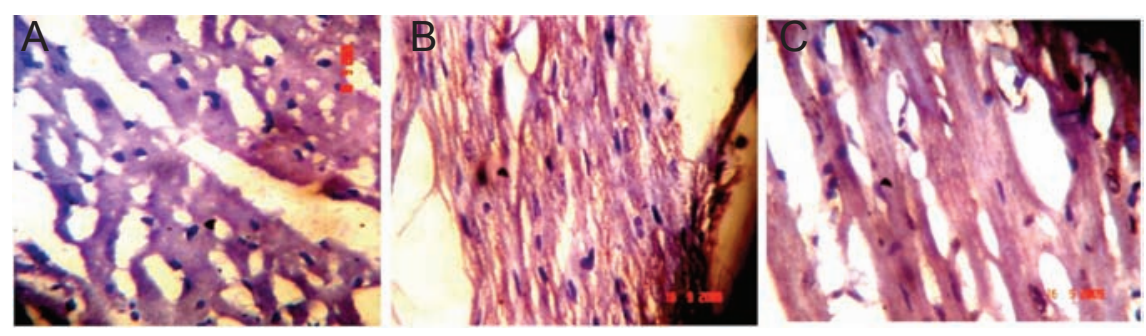

Figure 3. Immunohistochemical findings of HSP70 proteins. A) Non-ischemic myocardium of sham group was weakly positive for HSP 70. B) Upregulation in the expression of HSP 70 as indicated by dark brown positive immunoreactivity is evident in the $T t(2.5 \mathrm{mg} / \mathrm{kg})$ control group as compared to sham group C) In the $T t(10 \mathrm{mg} / \mathrm{kg})$ control groups, significant up regulation of HSP 70 expression as compared to sham group. Figures are representative of 6 separate experiments. 
addition, subsequent to ISP induced myocardial necrosis, Tt (2.5 and $10 \mathrm{mg} / \mathrm{kg}$ ) treatment decreased lipid peroxidation (reduced formation of TBARS from fatty acids). Previously, it has been reported that oral administration of methanolic fraction of Tt fruit extract at dose 6 $\mathrm{mg} / \mathrm{kg}$ body weight provided protection against the mercuric chloride induced lipid peroxidation in the mice. ${ }^{30}$ Saponins, the active constituent of Tt inhibit the metabolism of arachidonic acid via the cyclo-oxygenase and lipooxygenase pathways that generates reactive oxygen species; resulting in a decrease in the levels of lipid peroxides.13,31 Furthermore, protection against ISP induced oxidative stress in Tt treated rat hearts was evidenced by preservation of endogenous antioxidants enzyme SOD and GSHPx. However, Tt failed to significantly prevent the loss of GSH and CAT enzyme subsequent to ISP challenge. It appears that the major burden of neutralizing the ISP induced oxidative stress was borne by GSH and not by the antioxidant enzymes SOD and GSHPx.

$\mathrm{Tt}(2.5 \& 10 \mathrm{mg} / \mathrm{kg})$ doses significantly prevented myonecrosis subsequent to ISP induced myocardial necrosis via up regulating the expression of basal HSP 70, enhancing the endogenous antioxidant network of the myocardium and maintaining the antioxidant status. This adaptogenic property may contribute to its cardioprotective effect and strengthen the antioxidant defense mechanisms of the heart. Among the various doses evaluated in the present study, Tt at $2.5 \mathrm{mg} / \mathrm{kg}$ exhibited optimum cardioprotective activity.

\section{Conclusions}

The present study, for the first time demonstrates that Tt $(2.5 \mathrm{mg} / \mathrm{kg})$ offered significant protection against ISP induced myocardial necrosis through a unique property of enhancement of basal endogenous antioxidants, heat shock protein and antioxidant property without producing any cytotoxic effects.

\section{References}

1. Meyer M, Trost SU, Bluhm WF, et al. Impaired sarcoplasmic reticulum function leads to contractile dysfunction and cardiac hypertrophy. Am J Physiol Heart Circ Physiol 2001;280:H2046-52.

2. Rona G, Kahn DS, Chappel CI. Studies on infarct-like myocardial necrosis produced by isoproterenol: a review. Rev Can Biol 1963;22:241-55.

3. Noronha-Dutra AA, Steen EM, Woolf N.
The early changes induced by isoproterenol in the endocardium and adjacent myocardium. Am J Pathol 1984;114:231-9.

4. Brekhman II, Dardymov IV. New substances of plant origin, which increase nonspecific resistance. Annu Rev Pharmacol 1969;9:419-30.

5. Das DK, Engelman RM, Kimura Y. Molecular adaptation of cellular defences following preconditioning of the heart by repeated ischaemia. Cardiovasc Res 1993; 27:578-84.

6. Lei XL, Chiou GC. Cardiovascular pharmacology of Panax notoginseng (Burk) F.H. Chen and Salvia muiltiorrhiza. Am J Chin Med 1986;14:145-52.

7. Rege NN, Thatte UM, Dahanukar SA. Adaptogenic properties of six rasayana herbs used in Ayurvedic medicine. Phytother Res 1999;13:275-91.

8. Sammut IA, Jayakumar J, Latif N, et al. Heat stress contributes to the enhancement of cardiac mitochondrial complex activity. Am J Pathol 2001;158:1821-31.

9. Penna C, Perrelli MG, Raimondo S, et al. Postconditioning induces an anti-apoptotic effect and preserves mitochondrial integrity in isolated rat hearts. Biochim Biophys Acta 2009;1787:794-801.

10. Sun W, Li H, Yang SJ. A triterpene saponin from Tribulus terrestris attenuates apoptosis in cardiocyte via activating PKC signaling transduction pathway. J Asian Nat Prod Res 2008;10:39-48.

11. Achenbach H, Hübner H, Reiter M. New cardioactive steroid saponins and other glycosides from Mexican Tribulus cistoides. Adv Exp Med Biol 1996;404:357-70.

12. Seth SD, Jagadeesh G. Cardiac action of Tribulus terrestris. Indian $\mathrm{J}$ Med Res 1976;64:1821-5.

13. Guo Y, Shi DZ, Yin HJ, Chen KJ. Effects of Tribuli saponins on ventricular remodeling after myocardial infarction in hyperlipidemic rats. Am J Chin Med 2007;35:309-16.

14. Ohkawa H, Ohishi N, Yagi K. Assay of lipid peroxide in animal tissue by thiobarbituric acid reaction. Anal Biochem 1979;95:3518.

15. Moron MS, Depierre JW, Manmerik B. Level of glutathione, glutathione reductase and glutathione-s-transferase activity in rat lung and liver. Biochem Biophys Acta 1979;82:67-78.

16. Paglia DE, Valentine WN. Studies on the quantitative and qualitative characterization of erythrocyte peroxidase. J Lab and Clin Med 1967;2:158-61.

17. Mishra HP, Fridovich I. The oxidation of phenylhydrazine: superoxide and mechanisms. Biochemistry 1976;15 681-7.

18. Aebi H. Catalase. Methods of Enzymatic Analysis. Ed. Hans Elrich Bergmayer.
Edition II. Vol 2; 1974.

19. Lowry OH, Rosebrough NJ, Farr AI. Protein measurements with the Folin phenol reagent. J Biol Chem 1951;193:265-75.

20. Lamprecht W, Stan F, Weisser H, Heinz F. Determination of creatine phosphate and adenosine triphosphate with creatine kinase. In: Bergmeyer HU, Ed. Methods of Enzymatic Analysis. New York, NY: Academic Press; 1974. pp.1776-1778.

21. Rastogi T, Reddy KS, Vaz M, et al. Diet and risk of ischemic heart disease in India. Am J Clin Nutr 2004;79: 582-92.

22. Reddy KS. Cardiovascular disease in India. World Health Statistics 1993;46:101-7.

23. Wang $\mathrm{P}$, Chen $\mathrm{H}$, Qin $\mathrm{H}$, et al. Overexpression of human copper, zincsuperoxide dismutase (SOD1) prevents postischemic injury. Proc Natl Acad Sci USA 1998;95:4556-60.

24. Marczin N, El-Habashi N, Hoare GS, Bundy RE, Yacoub M. Antioxidants in myocardial ischemia-reperfusion injury: therapeutic potential and basic mechanisms. Arch Biochem Biophys 2003;420:222-36.

25. Yim MB, Chock PB, Stadtman ER. Copper, zinc superoxide dismutase catalyzes hydroxyl radical production from hydrogen peroxide. Proc Natl Acad Sci USA 1990;87:5006-10.

26. Hong Yu, Munehiro Yokoyama, Goro Asano. Time Course of Expression and Localization of Heat Shock Protein 72 in the Ischemic and Reperfused Rat Heart. Jpn Circ J 1999;63:278-87.

27. Tanwar V, Sachdeva J, Golechha M, et al. Curcumin protects rat myocardium against isoproterenol-induced ischemic injury: attenuation of ventricular dysfunction through increased expression of Hsp27 along with strengthening antioxidant defense system. J Cardiovasc Pharmacol 2010;55:377-84.

28. Peng W, Zhang $\mathrm{Y}$, Zheng $\mathrm{M}$, et al. Cardioprotection by CaMKII-deltaB is mediated by phosphorylation of heat shock factor 1 and subsequent expression of inducible heat shock protein 70 . Circ Res. 2010;106:102-10.

29. Kim SC, Stice JP, Chen L, et al. Extracellular heat shock protein 60 , cardiac myocytes, and apoptosis. Circ Res 2009;105:1186-95. Epub 2009 0ct 29.

30. Kavitha AV, Jagadeesan G. Role of Tribulus terrestris (Linn.) (Zygophyllacea) against mercuric chloride induced nephrotoxicity in mice, Mus musculus (Linn.). J Environ Biol 2006;27:397-400.

31. Kumar M, Soni AK, Shukla S, Kumar A. Chemopreventive potential of Tribulus terrestris against 7,12- dimethylbenz (a) anthracene induced skin papillomagenesis in mice. Asian Pac $\mathrm{J}$ Cancer Prev 2006;7:289-94. 